\title{
Mobility Contrast Effect on Environment in Manet as Mobility Model
}

\author{
Tarkeshwari kaul $^{1}$, R.K.kapoor ${ }^{2}$ \\ ${ }^{1,2}$ (Department of computer science and application, NITTTR, INDIA)
}

\begin{abstract}
Manet is a type of ad-hoc network without any infrastructure and connects mobile nodes through wireless channels. It gives environment in which nodes can be connected dynamically as they enter the area. The area can be any place where nodes can move uniformly or randomly like disaster hitting places, shopping malls, airport, station, campus, business meeting, and temple etc. Depending on the terrene, and physical infrastructure and environment that may cause obstacle in the movement of nodes in the given area, the mobility pattern of nodes is different. These involve the mobility environment as major factor for mobile nodes while sharing information in mobile ad-hoc network. The characteristics of mobility of nodes in Manet are application based and have significant impact on the performance of routing protocols. While studying the performance of MANET routing protocols, environment parameter as mobility is important and can be considered remarkably so that realistic scenario can be constructed for the accurate analysis of performance of routing protocols.
\end{abstract}

Keywords - Mobile ad-hoc network (Manet), Mobility Models, and Routing protocols.

\section{Introduction}

Manet is a collection of mobile nodes connect together to form a distributed network, where medium is wireless with no base station. Manet topology is unpredictable and changes rapidly. In Manet, mobile nodes work in two forms; as a node to send and receive packet as well as a router to route a packet. Nodes movement in Manet environment is mainly random, and the uniform movement is seen rare. Manet mainly used in areas where infrastructure based communication cannot be used such as disaster hit areas, mall, campus, airport, station, business meetings etc. It is a temporary network without wires and administration intervention. While studying the performance of various routing protocols in MANET, mobility pattern of nodes has to be taken in consideration so that routing protocol can cope up with node movement which is responsible for dynamic network topology. For the dynamic topology in Manet, the routing protocols behavior has to be analyzed using varying node speed, traffic and network size. But one of the most important is the mobility pattern of nodes.

The organization of the paper is as follows. Section 2 provide the information of major Mobile Ad hoc routing protocols. Section 3 covers different mobility patterns based on their properties. Section 4 present factors affecting mobility environment. Section 5 shows the effect of routing protocols in different environments and finally conclusion, future work in section 6 and followed by references.

\section{Routing Protocols}

Nodes in the network connected by wireless links which is less reliable media as compared to wired media hence the routing in such network is bit complex. Nodes are moving randomly which bring frequent topology change so it is a challenging task for routing process. Routing protocols play an important role for routing Process. In Manet there are two main approaches for routing.

\subsection{Proactive}

This approach is table driven work by using periodic protocols. This means that each node contain information of their neighbors in table form in which routing information is updated at a particular interval. The proactive routing protocols are independent of whether or not the route is needed [8]. Control messages are transmitted with periodically intervals. Even if there is no data flow still control messages are transmitted. Routing protocols come under this approach are DSDV, OLSR etc. some advantages are route discovery is not required and low latency for real time applications. Disadvantages are unused paths occupy a significant part of the available bandwidth.

\subsubsection{Destination Sequenced Distance Vector (DSDV)}

DSDV is a pro-active routing protocols based on bellman-ford algorithm. It contains the feature of distance- vector algorithm. Each node contain routing table which contain information of only next-hop for possible destination. Whenever any change take place in network immediately updates have to be transmitted. As soon as topology changes, destination assigns Sequence numbers and this number is used by source to send 
the next update. Each node uses 2 mechanisms to send out the DSDV updates. They are, Periodic Updates and triggered updates [1] [2.]

\subsubsection{Optimized Link State Protocol (OLSR)}

OLSR is a proactive protocol based on link state protocol. It is an IP routing protocol optimized for mobile ad-hoc networks and uses hello and topology control (TC) messages to discover and then disseminate link state information throughout the mobile ad-hoc network. Individual nodes use this topology information to compute next hop destinations for all nodes in the network using shortest hop forwarding paths. Since OLSR is proactive protocol, the routes to all destinations within given network are known and maintained before use. This phenomena is useful for some systems and network applications as there is no route discovery delay associated with finding a new route [3]

\subsection{Reactive}

This approach is on-demand or source initiated. The name itself shows it is a re-active means for node to send information to other node in the network need to find the path first. In order to reduce traffic in network route is discovered. Routing protocols come under this approach are AODV, TORA, DSR etc. some advantages are to update routing table not require periodic flooding the network. Flooding requires when it is demanded and Beaconless so it saves the bandwidth. Disadvantages are for route finding latency is high and Excessive flooding of the network causes disruption of nodes communication.

\subsubsection{Ad-Hoc on Demand Distance Vector Routing (AODV)}

AODV is a reactive protocol. Discover route is one of the plus point of this protocol it is use in unicast and multicast of data packets. It uses a destination sequence number .AODV enables multihop routing between the mobile nodes to establish a network or to maintain a network. AODV is a distance vector algorithm whose work is to discover and maintain links through different messages. When one node wants to connect with other node it broadcasts a Route Request to all its neighbors. The route request propagates throughout the network until it reaches the destination. When it reach to destination then it unicast a request reply back to the source. AODV uses Hello message information to know the availability of the neighbor nodes [4]

\subsubsection{Dynamic Source Routing (DSR)}

DSR is a reactive protocol which works on two phase's route discover and maintain. Route discovery is a dynamic process to send packet to destination through multiple hops. DSR protocol utilizes the source routing $\&$ maintains active routes. Each packet header contains the ordered node list of the path to destination called source routing. DSR doesn't include periodic updating by sending messages, therefore reduce bandwidth overhead, avoid large update throughout Manet network, and conserve battery power. DSR depend on MAC layer for support [5.][6]

\subsubsection{Temporally - Ordered Routing Algorithm (TORA)}

TORA protocol is a reactive protocol based on link reversal. It works by establishing the temporal order for topological changes. Multiple Routes are created from source to destination as per requirement. It reduces the effect of dynamic topological. In this protocol node need to maintain only the information of neighbor nodes. This protocol has three functions for packet delivery Query, Update and Clear. Query packets are used for creating packet, update is use for maintaining routes and clear packet is use for routes deduction. TORA is scalable and adaptive routing protocol. TORA uses metric for route discovery and route maintenance. This metric create a directed cyclic graph (DAG) at the destination. TORA is a single-pass procedure with the assumption that all the nodes have synchronized clocks to create topological change events [7][2]

\section{Mobility Models}

The mobility model is designed to describe the movement pattern of mobile nodes, and how their location, velocity and acceleration change over time. Mobility models are important for studying node behaviour in given environment. Mobility models can be differentiated according to their dependencies, restriction, movement etc. different model are having different movement pattern and form different characteristics thus important in analysing the performance of routing protocol in Manet.

\subsection{Random Mobility Models}

Random models are free from dependencies and restrictions. These models play an important role in reflecting the realistic traveling pattern of the nodes through various parameters. The following models come under these categories. 


\subsubsection{Random Waypoint Mobility Model}

This is most popular mobility model in Manet. Whenever change in speed or direction take place, nodes wait in a location for particular time interval. When this time expires, Mobile nodes randomly choose destination with speed in between [min, max]. Waypoint model contain the spatial dependency. Nodes movement is non-uniform. Neither a closed-form expression of this distribution nor an in-depth investigation found. These facts make it difficult to make simulation to find the accurate performance analysis. In this model, nodes timeslot is arbitrary for each movement and few nodes may remain stationary for the entire simulation time $[8][11]$

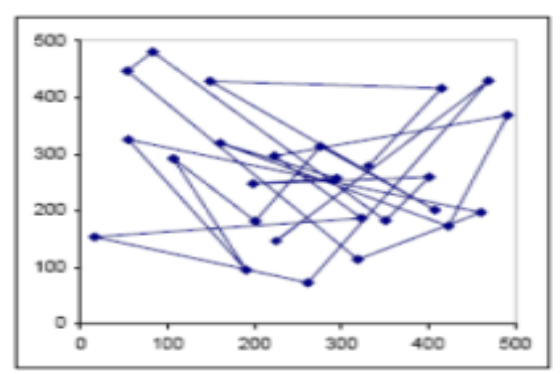

Fig.1 Random waypoint mobility model

\subsubsection{Random Walk Mobility Model}

Random Walk is a memory-less mobility pattern. This characteristic can generate unrealistic movements such as sudden stops and sharp turns. In this mobility model, mobile nodes travel from one location to another in random direction and wit random speed .the new speed should be [min-speed, max-speed] and [0, $2 *$ pi] respectively. Each movement in this Mobility Model is either a constant time interval or a constant traveled distance; at the end of each movement new direction and speed are calculated. Since many nodes movement in nature is unpredictable, this Mobility Model was developed to cope up with this erratic movement. If the moving nodes in this model reach a simulation boundary then according to this mobility model, it bounces off the simulation border with an angle determined by the incoming direction. Mobility model on a 1-Dimension or 2-dimension surface returns to the origin with complete certainty. This shows that the random walk mobility model is use to test the mobility of nodes around the starting point without concern about its reachable point [10]

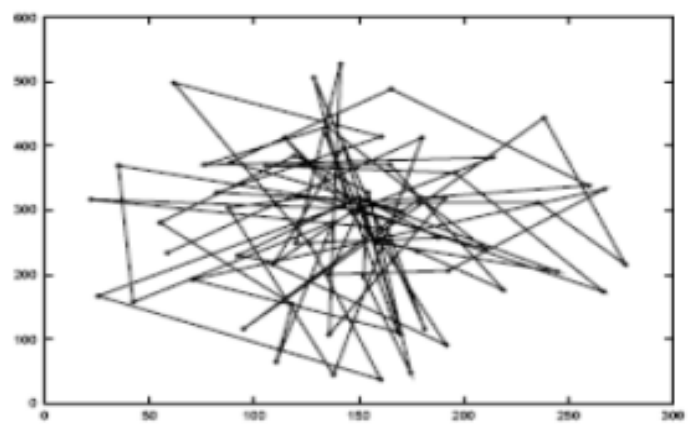

Fig. 2 Random walk mobility model

\subsubsection{Random Direction Mobility Model}

In Random direction mobility model direction of mobile nodes is similar to random walk mobility model. mobile nodes moves randomly throughout the network but when nodes reach to the boundary it pause there for specific time afterward it choose some random direction and continue the process. To change direction, nodes are not forced to travel to the simulation boundary. The main purpose of Random Direction Mobility Model is to overcome with the clustering of nodes in one part of simulation area. Contrast to random direction mobility model, in Random Waypoint Mobility Model cluster occurs near the center of the simulation area. In the Random direction Mobility Model, it is not require for nodes to pass through center of simulation area when node chose a new destination to travel but the probability is high in waypoint model. [2] 


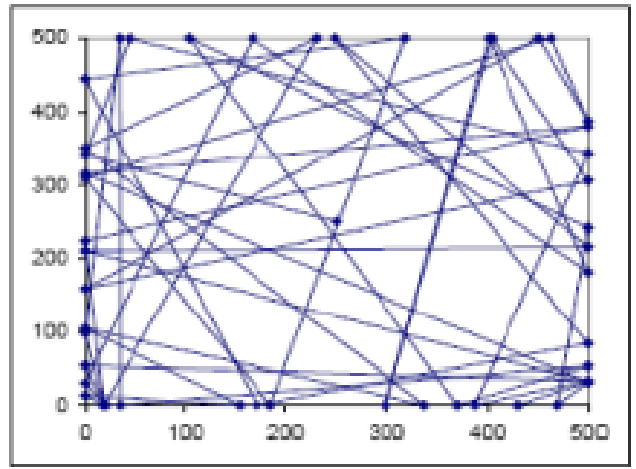

Fig. 3 Random direction mobility model

\subsection{Group Mobility Models}

\subsubsection{Referential Point Group Mobility Model (RPGM)}

RPGM is design as a cluster of similar nodes. Each cluster has a cluster head i.e. group leader which determines the group's motion behavior. Initially, each node of the group is uniformly distributed around their group leader. Further group leader randomly derive speed and direction for every node in other words decide group mobility. All nodes depend on group leader to travel. This model has high special dependence. The movement in group mobility can be characterized as follows:

a) Vmember $(\mathrm{t})=$ Vleader $(\mathrm{t})+$ random ()$\_S D R \_$max speed

b) member $(\mathrm{t})=$ leader $(\mathrm{t})+\operatorname{random}()_{-}$ADR _ max angle

Where $0<(\mathrm{SDR}, \mathrm{ADR})<1, \mathrm{SDR}=$ Speed Deviation Ratio and ADR $=$ Angle Deviation Ratio.

SDR and ADR are used to control the deviation of the velocity of group nodes from that of the leader. Angle and speed as a max speed and max angle are used to specify the maximum deviation a group member can hold [9][11]

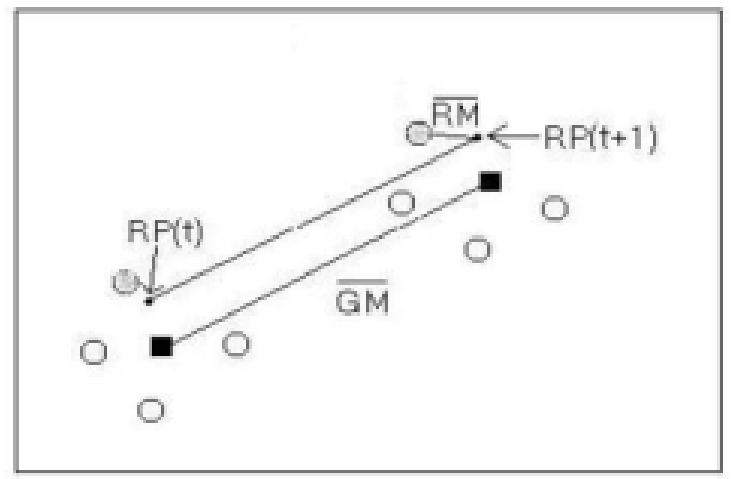

Fig.4 Referential point group mobility Model

\subsubsection{Nomadic Community Mobility Model}

The Nomadic Mobility Model is used to represent the group motion scenario where number of nodes move together randomly from one location to another. This model based on reference grid which is determined by the movement of group nodes. Inside the group, each node can offset some random vector to its predefined reference point.

Formally, new_pos = ref_pos + random_vector

Where is a small random vector used to offset the movement of mobile node. Nomadic Community Mobility Model shares the same reference grid. Furthermore, the movement in the Nomadic Community Model is erratic. [12] 


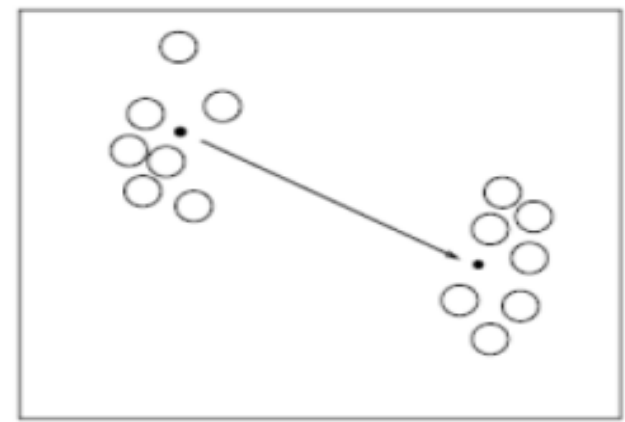

Fig. 5 Nomadic community mobility model

\subsubsection{Column Mobility Model}

In column Mobility Model mobile nodes are moving forward randomly in group around a line. Mobile nodes movement is random around the grid and new grid is moved by predefined offset which is same for all nodes calculated using the random distance and angle .this model specify the nodes movement pattern in two form one is perpendicular and another is parallel. Column mobility model is similar to nomadic community mobility model except that in this model each column has its own reference point. The movement of nodes is constant in Column Mobility Model. New reference grid is obtained from following formula

Formally, New_grid $=$ old_grid + advance vector

Here advance vector is the predefined grid [12][13]

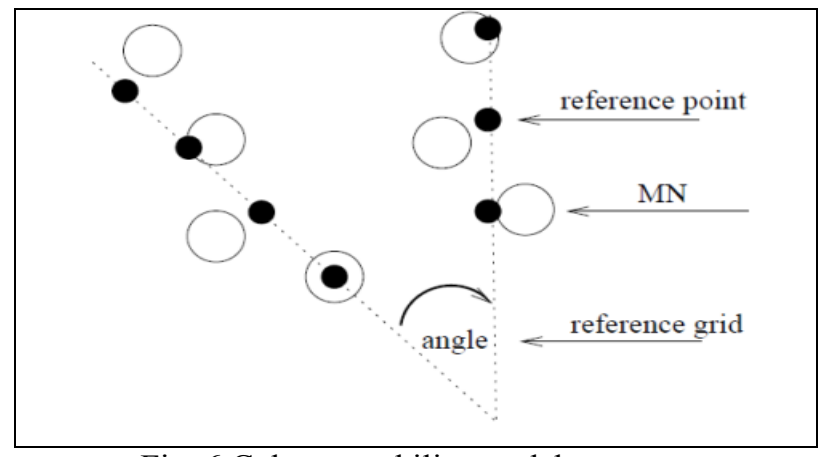

Fig. 6 Column mobility model

\subsubsection{Pursue Mobility Model}

The Pursue Mobility Model emulates scenarios where single node is capture by several other nodes. Node movement is based on the random waypoint model but the randomness is limited inorder to track the target node effectively. Seeker nodes move toward the target node with same speed. It is also a part of group mobility model where group of nodes movement is taking place.

Formally, new_position $=$ old_position + vt (positiontarget-old_position) + random vector

Where the expected position of node is targeted node being pursued at time $t$ and is a small random vector used to offset the movement of mobile node. The main purpose of this node is in target tracking and law enforcement [12]

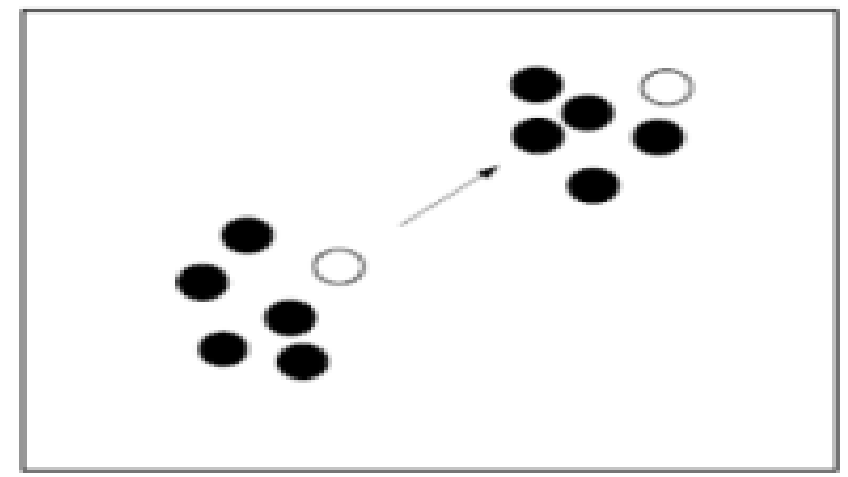

Fig. 7 Pursue mobility model 


\subsection{Map Based Mobility Models}

\subsubsection{Manhattan Mobility Model}

Manhattan model to emulate the movement of nodes on streets defined by maps. The map is composed of horizontal and vertical streets. Each street has two lanes crossing to each other (vertical streets, horizontal streets). The mobile nodes are permitted to move in any alternative direction along the grid on the map. At the intersection point of two lanes, the mobile node can go in any direction according to their choice either left or right or straight. Path Choice is probabilistic, same street probability is 0.5 , left turning probability is 0.25 and right turning probability is 0.25 . Velocity of the nodes depends on the previous time slot. Node velocity is also limited by the velocity of the preceding node on the same lane. This mobility model has high spatial and temporal dependence. It imposes restrictions on node mobility. SDR and ADR are two main parameters of Manhattan models [8][11]

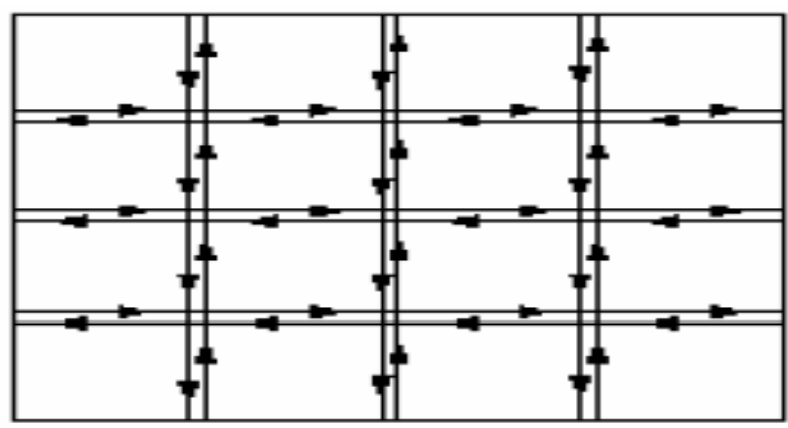

Fig. 8 Manhattan mobility model

\subsubsection{Freeway Mobility Model}

Freeway mobility model is use to imitate the motion activities of mobile nodes using maps. The map composed of number of freeways and each have lanes in both directions. Each lane restricts mobile nodes on the freeway. If two nodes on the same freeway lane at a Safety Distance, then the velocity of the following node cannot exceed the velocity of preceding node. The velocity of node is temporally dependent on its previous velocity. Inter-node and intra-node are related to each other. Due to these relationships, the Freeway mobility model has spatial dependence and high temporal dependence. It also imposes restrictions on the nodes movement by not allowing nodes to change its lane.it are similar to the Manhattan mobility model but nodes movement is restricted in freeway model [11]

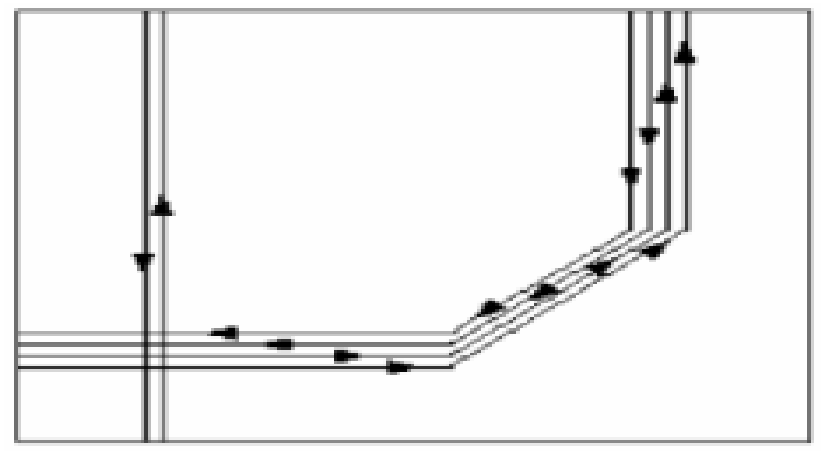

Fig. 9 Freeway Mobility Model

\subsection{Particle Based Mobility Models}

\subsubsection{Pedestrian Mobility Model}

This mobility model mainly use for small area and areas which can be cover by walk. In this type of model destination is usually fixed it is developed in concern to the people who are covering area by walk. It is use in awareness of persons for their own safety, help them to take proper decision and guide them. Some example of this type of model is seen while crossing road, in analyzing signal, moving in any shopping mall, bus station etc. it also help users in accessing the priority places. It has temporal dependencies between nodes. Nodes movement is pedestrian form where speed and direction are limited. Model created with pedestrian, pedestrians move in continuous space, reacting on different kinds of obstacles and other pedestrians [13][14] 


\subsection{Memory Based Mobility Models}

\subsubsection{Gauss-Markov Mobility Model}

The Gauss-Markov Mobility Model works on timeslot i.e. different level by one parameter. At stating each node is assign a particular speed and direction which is keep on changing at a particular interval of time. Mobility model calculate the new speed and direction of node by using speed and direction of previous node.

$$
V_{\mathrm{n}}=\beta \mathrm{V}_{\mathrm{n}-1}+(1-\beta) \Omega+\sqrt{(1-\beta)^{2}} \mathrm{x}_{\mathrm{n}-1}
$$

Where $\beta(0 \leq \beta \leq 1)$ is tuning parameter for varying randomness, $\Omega$ is a constant representing the mean value of speed and direction as $n \rightarrow \infty, x n-1$ is a random variable from a Gaussian distribution

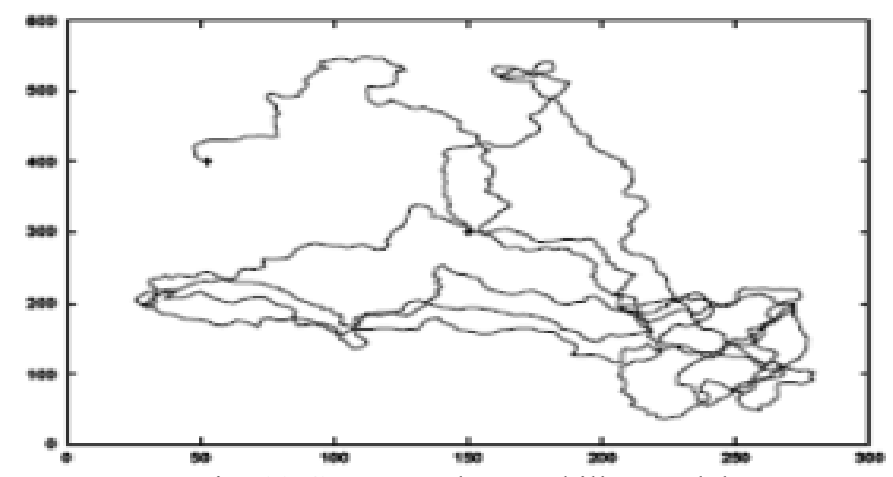

Fig. 10 Gauss-Markov mobility model

In the Gauss-Markov model, the temporal dependency plays a key role in determining the mobility behavior. In by emulating the mobility behavior of users in real life, it is also observed that the temporal dependency is an important mobility characteristic that should be captured. The Gauss-Markov Mobility Model has some memory so it knows where to stop and turn. [8][10]

\subsubsection{Boundless Simulation Area Mobility Model (BSAMM)}

In BSAMM, speed and direction of current node depends on the speed and direction of preceding node. Both speed and direction are updated at particular interval of time. When a node reaches one side of the boundary, it does not stop but continues its movement and go to opposite side of the area in same direction and with same speed. When time interval finish, the node chooses a new direction and speed and continues its movement. It has temporal dependency.

Formally,

$$
\begin{gathered}
v(t+\Delta t)=\min \left[\max (v(t)+\Delta v, 0), v_{\max }\right] \\
\theta(t+\Delta t)=\theta(t)+\Delta \theta \\
x(t+\Delta t)=x(t)+v(t) * \cos \theta(t) \\
y(t+\Delta t)=y(t)+v(t) * \sin \theta(t)
\end{gathered}
$$

Where $v_{\max }$ is the maximum velocity defined in the simulation, $\Delta \mathrm{v}$ is the change in velocity which is uniformly distributed between [-Amax $* \Delta \mathrm{t}, A \max * \Delta \mathrm{t}$ ], Amax is the maximum acceleration, $\Delta \theta$ is the change in direction which is uniformly distributed between $[-\alpha * \Delta \mathrm{t}, \alpha * \Delta \mathrm{t}]$, and $\alpha$ is the maximum angular change in the direction. [12]

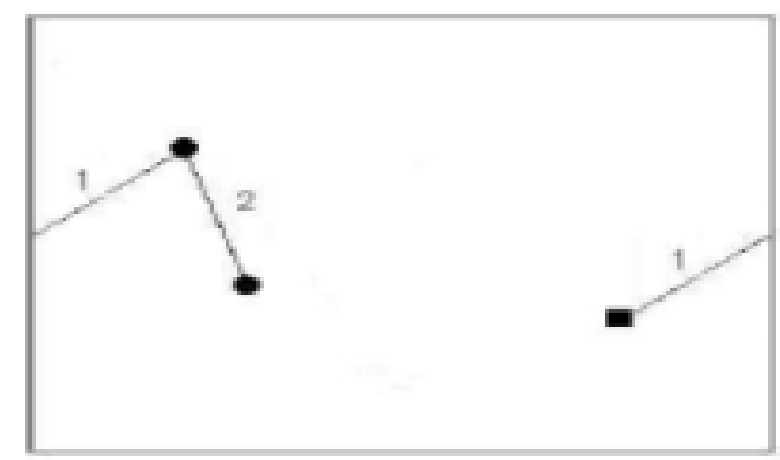

Fig. 11 Boundless simulation area Mobility Model 


\subsubsection{Smooth Mobility Model}

Smooth mobility model node speed depends on the time slot of previous node. It shows the temporal dependency of speed. It is also found that it result in more realistic movement behaviors. Instead of the sharp turn and sudden acceleration or deceleration, it also proposes to change the speed and direction of node movement incrementally and smoothly. It is observed that mobile nodes in real life tend to move at certain preferred speeds rather than at speeds uniformly distributed in the range [min-speed, max-speed]. Therefore, in Smooth Mobility model, the probability distribution of node speed has a high probability, while a uniform distribution is assumed on the remaining part of entire interval. In Smooth Mobility Model, Poisson process is working in which the frequency of speed changes. Upon an event of speed change, a new target speed is chosen according to the probability distribution function. Then, the speed of mobile node is changed incrementally from the current speed to the targeted new speed by acceleration speed or deceleration speed. [10]

\subsection{Heterogeneous Mobility Models}

These types of mobility models are the combination of two or more type of mobility model. By combination be indicate that properties of more than one models are inherited like entity mobility model and group mobility model. Based on the requirement of more real time scenario we can configure a heterogeneous mobility model.

\section{Factors Affecting Mobility in Manet}

The framework states that a realistic mobility model should include:

\subsection{Real based topological maps}

Such maps should manage different densities of roads, contains multiple lanes, different categories of streets, buildings and associated velocities.

\subsection{Hindrance}

Wireless medium and mobility itself is big hindrance to find exact scenario.

\subsection{Attraction}

When source or destination is random then network effect more to attraction points.

\subsection{Simulation}

Traffic can take place between any number of nodes we cannot fix exact scenario for it.

\subsection{Random movement}

Mobile nodes mobility is not uniform. In real life scenario, it is not easy to predict the movement because user is much affected by attraction points. Time configuration is required in many terrain areas.

\subsection{Battery power}

Mobile nodes battery power is not fixed and limited.

V. Effect of Routing Protocol in Different Environment

\begin{tabular}{|c|c|c|c|c|}
\hline Model & AREA & $\begin{array}{c}\text { ROUTING } \\
\text { PROTOCOL }\end{array}$ & EXAMPLE & SIMULATOR \\
\hline Random & rural & proactive & park & opnet, qualnet \\
\hline Group & urban, rural & $\begin{array}{c}\text { proactive, } \\
\text { reactive }\end{array}$ & $\begin{array}{c}\text { battlefield } \\
\text { omnet }\end{array}$ \\
\hline Map & urban & reactive & street & opnet \\
\hline Memory & urban, rural & $\begin{array}{c}\text { reactive, } \\
\text { proactive }\end{array}$ & law enforcement & opnet \\
\hline Particular & urban, rural & $\begin{array}{c}\text { proactive, } \\
\text { reactive }\end{array}$ & road crossing & qualnet \\
\hline
\end{tabular}

\section{Conclusion}

This paper contains the overview of mobility models available in research of mobile Ad Hoc Networks. These mobility models play a vital role in evaluating the performance of routing protocols. Today's trend is to go toward the more realistic scenario in terms of mobile mobility so mobility models with varying properties are illustrated so that the complex motion of nodes can be capture to analyze the performance more accurately. 
Several simulators are available to analyze within mobility model but each categories of mobility models are not supported by all.

In Future, More work is to be done in this domain in order to increase the realism. Further properties need to be analyzed. More work is to be done on Routing protocol which can support realism and give rise more reliable next generation networks. In order to study the performance of routing protocols using different mobility model a study is being carried out as our future work. The result of the study would be useful to analyze the performance of various routing protocols in different mobility pattern.

\section{References}

[1] Sabina Baraković, Suad Kasapović, and Jasmina Baraković, "Comparison of MANET Routing Protocols in Different Traffic and Mobility Model”, Telfor Journal, Vol. 2, No. 1, 2010

[2] M.K.Jeya Kumar and R.S.Rajesh, "Performance Analysis of MANET Routing Protocols in different mobility models", IJCSNS International Journal of Computer Science and Network 22 Security, VOL.9 No.2, February 2009.

[3] E. Hyytiä, H. Koskinen, P. Lassila, A. Penttinen and J. Virtamo "Random Waypoint Model in Wireless Networks"

[4] Youssef Saadi, Said El Kafhali, Abdelkrim Haqiq, Bouchaib Nassereddine, “ Simulation Analysis of Routing Protocols using Manhattan Grid Mobility Model in MANET”, International Journal of Computer Applications (0975 - 8887) Volume 45- No.23, May 2012

[5] Joy Ghosh, Sumesh J. Philip, Chunming Qiao, "Performance Analysis of Mobility Based Routing Protocols in MANET”, UB CSE 2004

[6] Per Johansson, Tony Larsson, Nicklas Hedman, Bartosz Mielczarek Mikael Degermark, ”Scenario-based Performance Analysis of Routing Protocols for Mobile Ad-hoc Networks", Mobicom '99 Scattlc Washington USA.

[7] Narinderjeet Kaur, Maninder Singh, “Caching Strategies in MANET Routing Protocols”, International Journal of Scientific and Research Publications, Volume 2, Issue 9, September 20121 ISSN 2250-3153 www.ijsrp.org

[8] Natarajan Meghanathan, "A Simulation Study on the Impact of Mobility Models on the Network Connectivity, Hop Count and Lifetime of Routes for Ad hoc Networks”, Informatica 34 (2010) 207-221

[9] Valentina Timcenko, Mirjana Stojanovic, Slavica Bostjancic Rakas, "MANET Routing Protocols vs. Mobility Models:Performance Analysis and Comparison", Proceedings of the 9th WSEAS International Conference on applied informatics and communications (AIC'09)

[10] Fan Bai, Ahmed Helmy, "A survey of Mobility Models in wireless Ad-hoc networks",

[11] Fan Bai, Ahmed Helmy, Narayanan Sadagopan, 'IMPORTANT: A framework to systematically analyze the Impact of Mobility on Performance of RouTing protocols for Adhoc NeTworks", IEEE infocom 2003

[12] Tracy Camp Jeff Boleng Vanessa Davies," A Survey of Mobility Models for Ad Hoc Network Research”, Wireless Communication \& Mobile Computing (WCMC): Special issue on Mobile Ad Hoc Networking.Research, Trends and Applications, vol. 2, no. 5, pp. 483-502, 2002.

[13] Michael Solomon Desta," A local Pedestrian Mobility Modelfor urban content sharing”10 jan 2010

[14] Jonghyun Kim, Stephan Bohacek, "A Survey-Based Mobility Model of People for Simulation of Urban Mesh Networks"

[15] Xiaoyan Hong, Mario Gerla, Guangyu Pei and Ching-Chuan Chiang, "A Group Mobility Model for Ad Hoc Wireless Networks".

[16] bhavyesh divecha; ajith abraham; crina grosan; sugata sanyal," impact of node mobility on manet routing protocols models “,journal of digital information management volume 5 number 1 february 2007.

[17] Gang Lu, Gordon Manson and Demetrios Belis, "Mobility Modeling in Mobile Ad Hoc Networks with Environment-Aware", journal of networks, vol. 1, no. 1, may 2006

[18] Ejiro .E. Igbesoko, Thaddeus Onyinye Eze, Mona Ghassemian, ” Performance Analysis of MANET Routing Protocols over Different Mobility Models", School of Computing and Mathematical Sciences University of Greenwich London, United Kingdom

[19] luc hogie, fr'ed'eric guinand, Gr'egoire Danoy Pascal Bouvry, Enrique Alba, "simulating realistic mobility models for large heterogeneous manets" 